\title{
On the Coextension of Cut-Continuous Pomonoids
}

\author{
David Kruml ${ }^{1} \cdot$ Jan Paseka ${ }^{1}$. Thomas Vetterlein ${ }^{2}$
}

Received: 9 June 2017 / Accepted: 11 July 2018 / Published online: 2 August 2018

(C) The Author(s) 2018

\begin{abstract}
We introduce cut-continuous pomonoids, which generalise residuated posets. The latter's defining condition is that the monoidal product is residuated in each argument; we define cut-continuous pomonoids by requiring that the monoidal product is in each argument just cut-continuous. In the case of a total order, the condition of cut-continuity means that multiplication distributes over existing suprema. Morphisms between cut-continuous pomonoids can be chosen either in analogy with unital quantales or with residuated lattices. Under the assumption of commutativity and integrality, congruences are in the latter case induced by filters, in the same way as known for residuated lattices. We are interested in the construction of coextensions: given cut-continuous pomonoids $K$ and $C$, we raise the question how we can determine the cut-continuous pomonoids $L$ such that $C$ is a filter of $L$ and the quotient of $L$ induced by $C$ is isomorphic to $K$. In this context, we are in particular concerned with tensor products of modules over cut-continuous pomonoids. Using results of M. Erné and J. Picado on closure spaces, we show that such tensor products exist. An application is the construction of residuated structures related to fuzzy logics, in particular left-continuous t-norms.
\end{abstract}

Thomas Vetterlein

Thomas.Vetterlein@jku.at

David Kruml

kruml@math.muni.cz

Jan Paseka

paseka@math.muni.cz

1 Department of Mathematics and Statistics, Masaryk University, Kotlářská 2, 61137 Brno, Czech Republic

2 Department of Knowledge-Based Mathematical Systems, Johannes Kepler University Linz, Altenberger Straße 69, 4040 Linz, Austria 
Keywords Partially ordered monoid - Cut-continuous pomonoid - Residuated poset · Coextension of cut-continuous pomonoids $\cdot$ Tensor product of modules over cut-continuous pomonoids $\cdot$ Closure space

\section{Introduction}

A map between posets is residuated if the inverse image of each principal ideal is again a principal ideal. A generalisation of this property has been studied in a number of papers: the so-called cut-continuous maps can be defined by the condition that the inverse images of principal ideals are just cuts, that is, sets consisting of the lower bounds of all their upper bounds $[1,5]$. The idea underlying the present work is to apply this definition in the context of pomonoids. For a pomonoid to be residuated means that the multiplication from the left or from the right is a residuated map. We relax this definition, just requiring that multiplication from the left or right is cut-continuous.

Cut-continuous pomonoids thus comprise residuated posets. Our actual interest concerns the latter structures and the starting point of considerations is the following. Let $L$ be a commutative, integral residuated lattice and let $C$ be a filter, that is, an upwards closed subalgebra of $L$. Then $C$ induces a congruence $\theta_{C}$ of $L$ and in fact all congruences of $L$ are of this form [2]. Let $P=L / \theta_{C}$ be the quotient; then we call $L$ a coextension of $P$ by $C$.

Given commutative, integral residuated lattices $P$ and $C$, we raise the question how to determine the coextensions of $P$ by $C$. It should be understood that this question is feasible only if $C$ is assumed to be of a particularly simple structure. The typical example is $C=\mathbb{R}^{-}$, the negative real cone endowed with the natural order and usual addition. In order to determine coextensions of the mentioned type, we may furthermore adopt what we could call a local viewpoint: we consider the monoidal multiplication of the enlarged structure restricted to single congruence classes. More specifically, let $L$ be as above, possessing the filter $C$. Then each congruence class is, first of all, a $C$-module: it is a lattice on which the elements of the residuated lattice $C$ act, and the action is in both arguments residuated. Second, let $R, S$ be two congruence classes such that $R=S \rightarrow(R \cdot S)$. Then the mapping $R \times S \rightarrow T,(r, s) \mapsto r \cdot s$, that is, the monoidal multiplication restricted to $R \times S$, is separately in each argument residuated and action preserving; we speak of a bimorphism of $C$-modules.

These simple observations imply that in order to construct a coextension of $P$ by $C$, we have to associate with each element of $P$ a $C$-module, and we have to determine bimorphisms in line with the product on $P$. The first step, the systematic determination of $C$-modules, does not seem possible in general. For a specific $C$, however, the possibilities can be very limited, an example being the mentioned negative real cone. But second, once the $C$-modules are given, the question is how to explore the bimorphisms. This is where the present paper intends to contribute.

Given two modules $R$ and $S$ over the residuated lattice $C$, a tensor product is a bimorphism to a further $C$-module $R \otimes_{C} S$, with the property that any other bimorphism factorises through it. Obviously, it would be valuable in the present context to have to our disposal a tensor product for any pair of modules over residuated lattices. Unfortunately, negative results dominate the scene. For instance, in the category of bounded posets and residuated maps between them, no tensor product exists [14].

Remarkably, the situation is different in the slightly broader context on which we actually focus in this paper. A tensor product does exist in the category of bounded posets and cutcontinuous maps [5]. This motivates us not to consider residuated lattices, but what we call 
cut-continuous pomonoids. The latter are more general than the former, but if the underlying poset is a complete lattice, both notions coincide. Moreover, on the basis of a suitable choice of morphisms, the construction of quotients resembles the case of residuated lattices.

We proceed as follows. We introduce in Section 2 the algebras under consideration - cutcontinuous pomonoids. In Section 3, we show that the Dedekind-MacNeille completion of any cut-continuous pomonoid exists and is a unital quantale. From Section 4 on, we focus on the analogy with residuated posets. We define morphisms generalising the case of residuated lattices and we show that quotients are induced by filters. In Section 5, we deal with an issue that is relevant for the sequel: the question when the restriction of a cut-continuous map is cut-continuous again. Section 6 is devoted to modules over cut-continuous pomonoids and we explain the usefulness of this notion in the framework of the coextension problem. In Section 7, we show that modules over cut-continuous pomonoids admit a tensor product. Here, we take benefit of the notion of a tensor product of closure spaces [9]. In Section 8, we demonstrate that our results may provide a framework for the determination of coextensions of cut-continuous pomonoids. In this context, we restrict to the case of a total order and we accordingly speak of cut-continuous tomonoids. The results hence apply in particular to residuated chains. In the final Section 9, some concluding remarks contain a summary of results, open questions and an outlook to the further research.

\section{Cut-Continuous Pomonoids}

For a subset $A$ of a poset $P$, we denote by $A^{\uparrow P}$ the set of its upper bounds and by $A^{\downarrow P}$ the set of its lower bounds. Usually, the poset $P$ will be clear from the context and we will simply write $A^{\uparrow}$ and $A^{\downarrow}$, respectively. The mapping $\mathcal{P}(P) \rightarrow \mathcal{P}(P), A \mapsto A^{\uparrow \downarrow}$ is a closure operator on $P$, that is, an extensive, monotone, and idempotent operation on the power set of $P$, which is ordered by set inclusion. Thus $P$ together with $\uparrow \downarrow$ is a closure space; we refer to [7] for an overview of the theory around this notion. The closure system associated with $\uparrow \downarrow$ is its image; we denote it by $\mathbf{M C}(P)$ and we call its elements cuts. That is, a cut is a set $A \subseteq P$ such that $A=A^{\uparrow \downarrow}$. Like any closure system, $\mathbf{M C}(P)$ is closed under arbitrary intersections and hence is with regard to the set-theoretical order a sup-lattice (that is, a complete lattice).

Note that $\mathbf{M C}(P)$ is generated by the principal ideals

$$
\{x\}^{\downarrow}=\{y \in P: y \leqslant x\}, \quad x \in P .
$$

Indeed, the cuts are the intersections of principal ideals, since $A^{\uparrow \downarrow}=\bigcap\left\{\{x\}^{\downarrow}: A \subseteq\{x\}^{\downarrow}\right\}$. Clearly, each cut is also the union of the principal ideals generated by its elements. Thus the set of all principal ideals is both meet-dense and join-dense in $\operatorname{MC}(P)$.

A map $\varphi: P \rightarrow Q$ between posets is called cut-continuous if $\varphi$ is continuous w.r.t. the closure operator $\uparrow \downarrow$, that is,

$$
\varphi\left(A^{\uparrow \downarrow}\right) \subseteq \varphi(A)^{\uparrow \downarrow}
$$

for any $A \subseteq P$. We follow here the terminology of [5], cf. also [6]; in [1], such maps are called closed. Equivalently, we may say that for any cut $B \subseteq Q$, the inverse image $\varphi^{-1}(B)$ is again a cut. In fact, $\varphi: P \rightarrow Q$ is cut-continuous if and only if the inverse image of each principal ideal is a cut.

A cut-continuous map $\varphi: P \rightarrow Q$ is monotone; in fact, $\varphi$ preserves all existing joins [1]. If $P$ and $Q$ are chains, $\varphi$ is cut-continuous if and only if $\varphi$ preserves all existing joins. Furthermore, if $P$ and $Q$ are sup-lattices, $\varphi$ is cut-continuous if and only if $\varphi$ preserves arbitrary joins. 
Given posets $A, B$, and $C$, we call a map $f: A \times B \rightarrow C$ separately cut-continuous if $f$ is cut-continuous in each argument.

We denote the category of posets and cut-continuous maps by $\mathcal{P C}$ and the full subcategories of bounded posets and of sup-lattices by $\mathcal{B P C}$ and $\mathcal{S U} \mathcal{P}$, respectively. Thus we have the inclusions

$$
\mathcal{S U P} \subseteq \mathcal{B P C} \subseteq \mathcal{P C}
$$

Let $e_{P}: P \rightarrow \mathbf{M C}(P)$ be the map assigning to each element of $P$ its principal ideal, that is, $e_{P}(x)=\{x\}^{\downarrow}$. Since $e_{P}^{-1}\left(\{A\}^{\downarrow}\right)=\left\{x \in P:\{x\}^{\downarrow} \in\{A\}^{\downarrow}\right\}=A$ for any $A \in \mathbf{M C}(P)$, we have that $e_{P}$ is a morphism of posets, which is moreover order-reflecting. In fact, the following is shown in [1, Thm. 2, Cor. 2], see also [5, Prop. 2.2]:

Proposition 2.1 The Dedekind-MacNeille completion $\mathrm{MC}$ gives rise to a reflector from the category $\mathcal{P C}$ as well as from $\mathcal{B P C}$ to the full subcategory $\mathcal{S U} \mathcal{P}$. The reflection morphisms are $e_{P}: P \rightarrow M C(P)$.

A partially ordered monoid, or pomonoid for short, is a monoid endowed with a compatible partial order. In this paper, we are concerned with pomonoids where the multiplication is separately cut-continuous.

Definition 2.2 A structure $(L ; \leqslant, \cdot, 1)$ is called a cut-continuous pomonoid if:

(C1) $(L ; \leqslant)$ is a poset;

(C2) $(L ; \cdot, 1)$ is a monoid;

(C3) the product $\cdot$ is separately cut-continuous, that is, the sets $\{z: y \cdot z \leqslant x\}$ and $\{z: z \cdot y \leqslant$ $x$ \} are cuts for any $x, y \in L$.

$L$ is in this case called commutative if so is the monoidal product. Moreover, $L$ is called integral if the monoidal identity 1 is the top element of the poset.

We note that cut-continuous pomonoids have already been investigated in a broader context in [10]. Following the terminology of [10], they are the $\mathcal{N}$-semigroups with identity, where " $\mathcal{N}$ " denotes the Dedekind-MacNeille completion.

Given a pomonoid $L$, we introduce for $x, y \in L$ and $S \subseteq L$ the left and right set residuals in $S$ :

$$
x / S y=\{z \in S: z \cdot y \leqslant x\} \text { and } y \backslash S x=\{z \in S: y \cdot z \leqslant x\} .
$$

Then $x / L y$ is the inverse image of the principal ideal $\{x\}^{\downarrow}$ under the right translation by $y$, and similarly, $y \backslash_{L} x$ is the inverse image of $\{x\}^{\downarrow}$ under the left translation by $y$. Note that the cut-continuity of $L$ can be expressed by saying that, for any $x, y \in L, x / L y$ and $y \backslash_{L} x$ are cuts.

Cut-continuous pomonoids can be viewed as a generalisation of residuated posets and a fortiori also of quantales. Indeed, by a residuated poset, we mean a pomonoid whose multiplication is in both arguments residuated. The latter condition in turn implies that, for any $x, y \in L, x / L y$ and $y \backslash_{L} x$ are principal ideals and hence cuts. We conclude that residuated posets are cut-continuous pomonoids.

Morphisms between residuated posets are commonly chosen such that not only the order and the monoidal structure is preserved, but also the residuals. On the other hand, morphisms between quantales are commonly defined differently, the preservation of arbitrary joins is required. Depending on whether we generalise the case of residuated structures or the case of quantales, cut-continuous pomonoids give rise to two distinct categories. We will discuss both of them in the subsequent Sections 3 and 4, respectively. 
Cut-continuous pomonoids share the following property with residuated posets. The lemma is immediate from the fact that cut-continuous maps preserve all existing joins.

Lemma 2.3 In a cut-continuous pomonoid, multiplication from the left or from the right distributes over existing joins.

We are especially interested in the case of a total order. Then the property that the multiplication distributes over existing joins even characterises cut-continuity.

A totally ordered monoid, or tomonoid for short, is a monoid endowed with a compatible total order. If the underlying order of a cut-continuous pomonoid is total, we will speak about "cut-continuous tomonoids".

Since a map between chains is cut-continuous if and only if all existing joins are preserved, we have the following proposition.

Proposition 2.4 Let $(L ; \leqslant, \cdot, 1)$ be a tomonoid. Then $L$ is cut-continuous if and only if multiplication from the left and from the right distributes over existing joins.

\section{Cut-Continuous Pomonoids and Quantales}

By a unital quantale, we mean a pomonoid such that its order is complete and multiplication distributes from both sides over arbitrary joins. As a reference for quantales, we may mention, e.g., $[13,15]$. Here, quantales will generally be assumed to be unital; "quantale" will henceforth mean "unital quantale".

Since quantales are always residuated, they can also be seen as complete residuated lattices. Quantales are obviously cut-continuous and from Lemma 2.3 we see that they actually coincide with those cut-continuous pomonoids that are sup-lattices.

In the present section, we will show that cut-continuous pomonoids can be completed to quantales and the completion gives rise to a reflector from a suitably defined category of cut-continuous pomonoids to the category of quantales.

For what follows, we recall that, with any monoid $(L ; \cdot, 1)$, we may associate the quantale $(\mathcal{P}(L) ; \subseteq, \cdot, 1)$; see, e.g., [15]. Here, we put

$$
X \cdot Y=\{x \cdot y: x \in X, y \in Y\}
$$

for $X, Y \subseteq L$. The residuals are given by

$$
\begin{aligned}
& Y \backslash X=\{z \in L: Y \cdot\{z\} \subseteq X\}, \\
& X / Y=\{z \in L:\{z\} \cdot Y \subseteq X\} .
\end{aligned}
$$

Theorem 3.1 Let $(L ; \leqslant, \cdot, 1)$ be a partially ordered monoid. The following conditions are equivalent:

(1) $(L ; \leqslant, \cdot, 1)$ is a cut-continuous pomonoid.

(2) $(\boldsymbol{M C}(L) ; \subseteq, \&, \mathbf{1})$ is a quantale, where

$$
X \& Y=(X \cdot Y)^{\uparrow \downarrow}
$$

for any $X, Y \in M C(L)$, and $\mathbf{1}=\{1\}^{\downarrow}$.

Proof (1) $\Rightarrow$ (2): This follows by [10, Prop. 2.2]. 
(2) $\Rightarrow(1)$ : Assume that $(\mathbf{M C}(L) ; \subseteq, \&, \mathbf{1})$ is a quantale. It suffices to show condition (C3) in Definition 2.3. But for any $x, y \in L$,

$$
\begin{aligned}
& \{z \in L: y \cdot z \leqslant x\}=\left\{z \in L:\{y\}^{\downarrow} \cdot\{z\} \subseteq\{x\}^{\downarrow}\right\}=\{y\}^{\downarrow} \backslash\{x\}^{\downarrow}, \\
& \{z \in L: z \cdot y \leqslant x\}=\left\{z \in L:\{z\} \cdot\{y\}^{\downarrow} \subseteq\{x\}^{\downarrow}\right\}=\{x\}^{\downarrow} /\{y\}^{\downarrow}
\end{aligned}
$$

are cuts.

Definition 3.2 A q-morphism $\varphi: L \rightarrow K$ between cut-continuous pomonoids is a cutcontinuous map preserving the monoidal structure. We denote by $q \mathcal{C C M}$ the category of cut-continuous pomonoids and q-morphisms and by $\mathcal{U Q}$ its full subcategory of (unital) quantales.

We recall that in $\mathcal{U} \mathcal{Q}$, q-morphisms are the standard morphisms of unital quantales, that is, maps preserving the monoidal structure and arbitrary joins.

The following proposition is an immediate corollary of [10, Prop. 2.5].

Proposition 3.3 The Dedekind-MacNeille completion MC gives rise to a reflector from the category $q \mathcal{C C M}$ to its full subcategory $\mathcal{U} \mathcal{Q}$. The reflection morphisms are $e_{L}: L \rightarrow$ $M C(L)$.

\section{Cut-Continuous Pomonoids and Residuation}

We will from now on view cut-continuous pomonoids as generalised residuated lattices. This requires some adjustment in regard to our choice of morphisms. Homomorphisms between residuated lattices are required to preserve the residuals and homomorphisms between complete residuated lattices do not in general preserve joins.

In the remainder of the paper, we will, unless otherwise stated, assume commutativity and integrality. By a "cut-continuous pomonoid" we will mean a "commutative, integral cut-continuous pomonoid".

For a subset $A$ of a poset $P$, we will denote by $\downarrow A$ the smallest downwards closed set containing $A$, that is, $\downarrow A=\{p \in P: p \leqslant a$ for some $a \in A\}$.

Definition 4.1 An r-morphism $\varphi: L \rightarrow K$ between cut-continuous pomonoids is a map preserving the order and the monoidal structure and such that, for any $x, y \in L$, we have:

$$
\downarrow \varphi(y / L x)=\varphi(y) / K \varphi(x) .
$$

We easily observe that this definition has the desired property. Indeed, assume that $\varphi: L \rightarrow K$ is a mapping between residuated posets $L$ and $K$ and that $\varphi$ preserves the order and the monoidal structure. Then we have $\downarrow \varphi(y / L x)=\downarrow \varphi(x \rightarrow y)$ and $\varphi(y) / K \varphi(x)=$ $\downarrow(\varphi(x) \rightarrow \varphi(y))$ for any $x, y \in L$, where $\rightarrow$ denotes the residuals of $L$ or $K$, respectively. Hence $\varphi$ is an r-morphism exactly if $\varphi$ preserves the residuum.

Furthermore, let $\varphi: L \rightarrow K$ be a surjective r-morphism. Then the partial order on $K$ is determined by the partial order on $L$ and the r-morphism $\varphi$ as follows: $y \leqslant z$ in $K$ if and only if there are $w, v \in L$ such that $w \leqslant v, y=\varphi(w)$, and $z=\varphi(v)$. Indeed, the "if" part is clear from the monotonicity of $\varphi$. To verify the "only if" part, assume that $y \leqslant z$ in $K$. Let $w^{\prime}, v \in L$ be such that $y=\varphi\left(w^{\prime}\right)$ and $z=\varphi(v)$. From $\varphi\left(w^{\prime}\right) \leqslant \varphi(v)$ it follows in view of Eq. 3 that $1 \in \downarrow \varphi\left(v / L w^{\prime}\right)$. But 1 is the top element of $K$, hence there is an element 
$a \in L$ such that $w^{\prime} \cdot a \leqslant v$ and $\varphi(a)=1$. Then the elements $w=w^{\prime} \cdot a$ and $v$ of $L$ have the desired properties.

Theorem 4.2 Cut-continuous pomonoids and r-morphisms between them form a category $r \mathcal{C C M}$.

Proof Obviously, the identity mappings are r-morphisms. Assume that $\varphi: L \rightarrow K$ and $\psi: K \rightarrow M$ are r-morphisms. Clearly, $\psi \circ \varphi$ preserves the order and the monoidal structure. Moreover, for $x, y \in L$, we compute

$$
\begin{aligned}
(\psi \circ \varphi)(y) / M(\psi \circ \varphi)(x) & =\psi(\varphi(y)) / M \psi(\varphi(x))=\downarrow \psi(\varphi(y) / K \varphi(x)) \\
& =\downarrow \psi(\downarrow \varphi(y / L x))=\downarrow(\psi \circ \varphi)(y / L x)
\end{aligned}
$$

and we conclude that $\psi \circ \varphi$ is an r-morphism as well.

A congruence $\theta$ of a cut-continuous pomonoid $L$ will be understood as being induced by a surjective r-morphism. We denote the quotient by $\left(L / \theta ; \leqslant \theta_{\theta},{ }_{\theta}, 1_{\theta}\right)$. Note that, for $a, b \in L$, we have $a / \theta \leqslant{ }_{\theta} b / \theta$ if and only if there are $a^{\prime} \theta a$ and $b^{\prime} \theta b$ such that $a^{\prime} \leqslant b^{\prime}$.

It turns out that congruences can be described in the same way as in the case of residuated lattices. As usual, a filter of a pomonoid means an upwards closed submonoid.

Theorem 4.3 Let $L$ be a cut-continuous pomonoid and let $\theta$ be a congruence on $L$. Then $C=1 / \theta$ is a filter. Moreover, for $x, y \in L$ we have $x \theta y$ if and only if there is a $c \in C$ such that $x \cdot c \leqslant y$ and $y \cdot c \leqslant x$. In particular, cut-continuous pomonoids are 1-regular.

Proof Let $K=L / \theta$ and let $\varphi: L \rightarrow K, x \mapsto x / \theta$ be the associated r-morphism. As $\varphi$ preserves the order and the product, $C=\varphi^{-1}(1)$ is a convex submonoid, that is, a filter.

Let $x, y \in L$ and assume $\varphi(x)=\varphi(y)$. By Eq. 3, we have $1 \in \downarrow \varphi(y / L x)$ and since 1 is the top element of $K$, it follows that $1 \in \varphi(y / L x)$, that is, there is an $a \in L$ such that $x \cdot a \leqslant y$ and $\varphi(a)=1$. In other words, there is an $a \in C$ such that $x \cdot a \leqslant y$. By a symmetric argument, there is also an $a^{\prime} \in C$ such that $y \cdot a^{\prime} \leqslant x$. Putting $c=a \cdot a^{\prime} \in C$, we have that $x \cdot c \leqslant y$ and $y \cdot c \leqslant x$.

Conversely, let $c \in C$ such that $x \cdot c \leqslant y$ and $y \cdot c \leqslant x$. Then $\varphi(c)=1$ and hence $1 \in \varphi(y / L x) \subseteq \downarrow \varphi(y / L x)=\varphi(y) / K \varphi(x)$. We conclude $\varphi(x) \leqslant \varphi(y)$. Similarly, we get $\varphi(y) \leqslant \varphi(x)$, and the second half of the assertion is shown as well.

We observe that any congruence on a cut-continuous pomonoid $(L ; \leqslant, \cdot, 1)$ is induced by a filter $C$ of $L$. We can hence identify a congruence $\theta$ with the filter $C=1_{\theta}=1 / \theta$ that induces $\theta$. We will refer in the sequel to the $\theta$-classes also as $C$-classes.

The situation concerning congruences of cut-continuous pomonoids is not as transparent as in the case of residuated lattices. We cannot establish a one-to-one correspondence between the congruences and the filters, as a filter does not necessarily induce a congruence. The following example is due to [8, Ex. 2].

Example 4.4 Let $L=\{(0, n): n \in \mathbb{N}\} \cup\left\{(1, z): z \in \mathbb{Z}^{-}\right\}$, where $\mathbb{Z}^{-}$are the negative integers (including 0$)$. We make $L$ into a chain by setting $(0, m) \leqslant(0, n)$ if $m \leqslant n,(0, n) \leqslant$ $(1, z)$ for any $n \in \mathbb{N}$ and $z \in \mathbb{Z}^{-}$, and $(1, y) \leqslant(1, z)$ if $y \leqslant z$. We define a product on $L$ as follows: let $(0, n) \cdot(0, m)=(0,0)$ for any $m, n \in \mathbb{N}$, and we let the product in all other cases be the smaller of the two elements. Then $(L ; \leqslant, \cdot,(1,0))$ is a cut-continuous tomonoid.

Consider now the filter $C=\left\{(1, z): z \in \mathbb{Z}^{-}\right\}$. The associated equivalence relation is a congruence of $L$ as a tomonoid. The quotient is (isomorphic to) the drastic tomonoid over 
the chain $\mathbb{N} \cup\{\infty$, where $\infty$ is the top element and the monoidal identity: all products are 0 except for those involving $\infty$. This tomonoid is evidently not cut-continuous.

We note that Theorems 4.2 and 4.3 extend to the category of commutative, integral pomonoids together with r-morphisms. Erné has shown that in this setting the desired oneto-one correspondence between the congruences and the filters does hold [8]. In addition, he has established a criterion that characterises the filters such that the quotients induced by them are cut-continuous.

\section{Restriction of Cut-Continuous Mappings}

Given a cut-continuous map $\varphi: P \rightarrow Q$ between posets, a restriction $\left.\varphi\right|_{S}: S \rightarrow Q$, where $S$ is a subposet of $P$, is not necessarily cut-continuous again. For example, let $[0,1]$ be the real unit interval endowed with the natural total order and consider the identity map on it, $\varphi:[0,1] \rightarrow[0,1], x \mapsto x$. Then the restriction of the map $\varphi$ to $\left[0, \frac{1}{2}\right) \cup\{1\} \rightarrow[0,1]$ is not cut-continuous. Similarly, let $[0,1] \cup[0,1]$ be the horizontal sum of two copies of $[0,1]$. Then the restriction of the identity map on $[0,1] \dot{\cup}[0,1]$ to the subset $\left[0, \frac{1}{2}\right) \dot{\cup}\left[0, \frac{1}{2}\right)$ is likewise not cut-continuous.

We shall present sufficient criteria for the restriction of cut-continuous maps to be cutcontinuous again. The results of this section are due to M. Erné [8] (cf. [4]). Since this paper might not be easily available, we will present the statements that we need together with proofs.

The central notion that we will use is cut-compatibility [8]. Namely, a subposet $S$ of a poset $P$ is called cut-compatible if

$$
A^{\uparrow s \downarrow S}=A^{\uparrow P \downarrow P} \cap S \text { for all non-empty } A \subseteq S .
$$

Note that $A^{\uparrow P \downarrow P} \cap S \subseteq A^{\uparrow s \downarrow s}$ always holds, but not in general the converse inclusion.

Furthermore, we will use the following slight relaxation of the notion of cut-continuity. Let $P$ and $Q$ be posets. A map $\varphi: P \rightarrow Q$ is called almost cut-continuous if the inverse image of each cut is either a cut or empty. A map is almost cut-continuous if and only if the inverse image of each principal ideal is a cut or empty. An almost cut-continuous map is monotone and preserves all existing non-empty joins.

Proposition 5.1 [8, Prop. 1] Let $S$ be a subposet of a poset $P$. Then the following are equivalent:

(1) S is cut-compatible.

(2) For any cut-continuous map $\varphi: P \rightarrow Q$, the restriction $\left.\varphi\right|_{S}: S \rightarrow Q$ is almost cutcontinuous.

(3) The restriction $\left.i d_{P}\right|_{S}: S \rightarrow P$ of the identity map $i d_{P}: P \rightarrow P$ is almost cut-continuous.

Proof (1) $\Rightarrow$ (2): Let $S$ be cut-compatible and let $\varphi: P \rightarrow Q$ be cut-continuous. Let $y \in Q$. By assumption, $A=\varphi^{-1}(\downarrow y)$ is a cut. Provided that $\left.\varphi\right|_{S}{ }^{-1}(\downarrow y) \neq \varnothing$, we get by cut-compatibility that $\left.\varphi\right|_{S}{ }^{-1}(\downarrow y)=A \cap S=A^{\uparrow P \downarrow P} \cap S \supseteq(A \cap S)^{\uparrow P \downarrow P} \cap S=(A \cap S)^{\uparrow s \downarrow S} \supseteq$ $A \cap S$, that is, $\left.\varphi\right|_{S} ^{-1}(\downarrow y)$ is a cut in $S$.

(2) $\Rightarrow$ (3): Evidently, $\operatorname{id}_{P}: P \rightarrow P$ is a cut-continuous map. Hence, assuming (2), we have that the restriction $\left.\operatorname{id}_{P}\right|_{S}: S \rightarrow P$ is almost cut-continuous. 
(3) $\Rightarrow$ (1): Let $\left.\operatorname{id}_{P}\right|_{S}: S \rightarrow P$ be almost cut-continuous. Let $A \subseteq S$ be non-empty and $b \in A^{\uparrow P}$. Evidently, $A \subseteq S \cap \downarrow b=\left.\operatorname{id}_{P}\right|_{S} ^{-1}(\downarrow b) \neq \varnothing$. Hence $\left.\operatorname{id}_{P}\right|_{S} ^{-1}(\downarrow b)$ is a cut in $S$, that is, $A^{\uparrow s \downarrow s} \subseteq(S \cap \downarrow b)^{\uparrow s \downarrow s}=S \cap \downarrow b$. It follows that $A^{\uparrow s \downarrow s} \subseteq A^{\uparrow P \downarrow P} \cap S$, that is, $S$ is cut-compatible.

We have the following two sufficient criteria for a subset of a cut-continuous pomonoid to be cut-compatible.

Lemma 5.2 [8, Lem. 1] Let L be a meet-semilattice and $S$ be a convex subset of L. Assume that either $L$ is totally ordered or $S$ has a greatest element. Then $S$ is cut-compatible.

Proof Let $A \subseteq S$ be non-empty. We have to show that $A^{\uparrow s \downarrow s} \subseteq A^{\uparrow L \downarrow L} \cap S$. Let $a \in A^{\uparrow s \downarrow s}$. This means that $a \in S$ and $a \leqslant q$ for any $q \in A^{\uparrow}$.

Assume first that $S$ has the greatest element $g$. Then, for any $r \in A^{\uparrow L}$, we have $r \wedge g \in$ $A^{\uparrow L}$ and since $A$ is a non-empty subset of the convex set $S$ it follows that $r \wedge g \in A^{\uparrow S}$ and thus $a \leqslant r$, that is, $a \in A^{\uparrow L \downarrow} \cap \cap S$.

Assume second that $L$ is totally ordered. Then, for $r \in A^{\uparrow L}$, we either have $r \in S$ and hence $a \leqslant r$. Or we have $r \notin S$; then it follows that $r \in S^{\uparrow L}$ by the convexity of $S$, and consequently $a \leqslant r$ also in this case. Thus $a \in A^{\uparrow L \downarrow_{L}} \cap S$.

In what follows we are interested in the question under which conditions the restriction of the multiplication in cut-continuous pomonoids to a pair of subsets remains separately cut-continuous. We note that this question was already studied in a more general context in [4] and [10].

The following theorem is a slight generalisation of [8, Thm. 1]. In this theorem as well as its corollary, commutativity and integrality need not be assumed.

Theorem 5.3 Let $R$ and $S$ be cut-compatible subsets of a cut-continuous pomonoid $L$, and let $T \subseteq L$ be such that, for all $x \in R, y \in S$, and $z \in T$, we have that $x \cdot y \in T$ and $z / R$, $x \backslash_{S} z$ are non-empty. Then the restricted multiplication $\left.\cdot\right|_{R \times S}: R \times S \rightarrow T$ is separately cut-continuous.

Proof Let us fix $y \in S$. The right translation by $y$, that is, the map $\varrho_{y}: L \rightarrow L, r \mapsto$ $r \cdot y$, is cut-continuous and $\left.\varrho_{y}\right|_{R}: R \rightarrow L$ is by Proposition 5.1 almost cut-continuous. By assumption, the range of $\left.\varrho_{y}\right|_{R}$ can safely be replaced by $T$ and we then still have that $\left.\varrho_{y}\right|_{R}: R \rightarrow T$ is almost cut-continuous. Furthermore, for any $z \in T, z / R$ y is non-empty for any $z \in T$, that is, the inverse image of $\{z\}^{\downarrow T}$ under $\left.\varrho_{y}\right|_{R}$ is non-empty. We conclude that $\left.\varrho_{y}\right|_{R}$ is even cut-continuous.

This shows that the multiplication $\left.\right|_{R \times S}: R \times S \rightarrow T$ is in the first argument cutcontinuous. We similarly argue for the second argument.

Corollary 5.4 Let $S$ be a cut-compatible subpomonoid of a cut-continuous pomonoid $L$ such that, for all $x, y, z \in S, z / s \quad y$ and $x \backslash_{S} z$ are non-empty. Then $S$ is a cut-continuous pomonoid as well.

\section{$6 C$-Modules and Coextensions}

We now introduce modules over cut-continuous pomonoids. Our definition is chosen in analogy with the case of modules over residuated lattices [17, Sec. 3.2]. 
We recall that all pomonoids are assumed to be commutative and integral. Also residuated posets will be understood to fulfil these properties.

Definition 6.1 Let $C$ be a cut-continuous pomonoid. Then a $C$-module is a poset $R$ together with an action $\star: C \times R \rightarrow R$ such that (i) $a \star(b \star x)=(a \cdot b) \star x$ and $1 \star x=x$ for any $a, b \in C$ and $x, y \in R$, and (ii) $\star$ is separately cut-continuous.

Moreover, a $C$-module $R$ is called order-transitive if for any $x, y \in R$ there is an $a \in C$ such that $a \star x \leqslant y$.

A morphism between $C$-modules $R$ and $S$ is a cut-continuous map $\varphi: R \rightarrow S$ such that $\varphi(a \star x)=a \star \varphi(x)$ for any $a \in C$ and $x \in R$. Finally, for $C$-modules $R, S$, and $T$, a bimorphism is a map $\psi: R \times S \rightarrow T$ that is a morphism of $C$-modules in each argument.

Note that a map $\psi: R \times S \rightarrow T$ being a bimorphism of $C$-modules means that $\psi$ is separately cut-continuous and preserves the action in each argument.

Given a congruence on some cut-continuous pomonoid, we are going to consider the action of the corresponding filter on single congruence classes.

Proposition 6.2 Let $L$ be a cut-continuous pomonoid and $\theta$ a congruence on L. Let $C=$ $1_{\theta}=1 / \theta$ be the filter inducing $\theta$, let $R, S \in L / \theta$ and $T=R \cdot{ }_{\theta} S$. Assume that either $L$ is $a$ tomonoid, or $L$ is a meet-semilattice and $R$ and $S$ possess top elements. Then the following holds.

1. Endowed with the inherited order and with the left multiplication by the elements of $C$, that is, with the action

$$
\star: C \times R \rightarrow R,(a, x) \mapsto a \cdot x,
$$

$R$ is an order-transitive $C$-module.

2. The multiplication, that is, the mapping

$$
R \times S \rightarrow T,(x, y) \mapsto x \cdot y,
$$

is a bimorphism from the $C$-modules $R$ and $S$ to the $C$-module $T$.

Proof Since $R, S$, and $C$ are convex, we conclude from Proposition 5.2 that these sets are cut-compatible.

(i) Clearly, $R=C \cdot{ }_{\theta} R$, $\star$ fulfils the associativity condition, and 1 acts neutrally.

Let $x \in C$ and $y, z \in R$. Since $y \theta z$, there is an $a \in C$ such that $a \cdot y \leqslant z$, i.e., the set $z / C y$ is non-empty. Similarly, since $a \cdot y \in R$ and $x \cdot(a \cdot y) \leqslant z$, also $x \backslash_{R} z$ is non-empty. From Theorem 5.3 we conclude that $\star$ is cut-continuous in both arguments.

Furthermore, the $C$-module $R$ is order-transitive by Theorem 4.3.

(ii) Let $x \in R, y \in S$ and $z \in T$. Since $x \cdot y \in T$ we get that $x \cdot y \theta z$. It follows that there is a $c \in C$ such that $(c \cdot x) \cdot y=x \cdot(c \cdot y)=c \cdot(x \cdot y) \leqslant z$, where $c \cdot x \in R$ and $c \cdot y \in S$. Therefore the sets $z / R \quad y$ and $x \backslash_{S} z$ are non-empty. Again by Theorem 5.3, the multiplication is in each argument cut-continuous.

The remaining assertions are immediate.

As an example in which Proposition 6.2 always applies, consider a congruence on a complete cut-continuous pomonoid such that the inducing filter is principal. Then all congruence classes possess a top elements and hence are $C$-modules. Indeed, let $c$ be the least 
element of the inducing filter $C$, let $R$ be some congruence class, and let $r \in R$. Then $x \cdot c \leqslant r$ and $r \cdot c \leqslant x$ for all $x \in R$, hence by cut-continuity $\bigvee R \cdot c \leqslant r$ and furthermore $r \cdot c \leqslant \bigvee R$, that is, $\bigvee R \in R$. Note that this remark covers the congruences on any finite residuated lattice, because filters are down-directed.

We have seen that we may form quotients of cut-continuous pomonoids by means of their filters. Let us now adopt the opposite viewpoint. For a cut-continuous pomonoid $K$, we call a further cut-continuous pomonoid $L$ a coextension of $K$ if there is a surjective r-morphism from $L$ to $K$, that is, if $K$ is (isomorphic to) a quotient of $L$. More specifically, if $C$ is the filter of $L$ inducing the quotient, we say that $L$ is a coextension of $K$ by $C$. The idea is to construct from $K$ and $C$ the coextensions of $K$ by $C$.

For given cut-continuous tomonoids $K$ and $C$, we are particularly interested in the construction of the totally ordered coextensions of $K$ by $C$. In particular, the $C$-classes are then chains.

To this end, it is useful to know all $C$-modules for a given underlying chain $R$ as well as the morphisms and bimorphisms between these $C$-modules. To substantiate this statement, we shall give an example from the realm of left-continuous t-norms.

A $t$-norm is a binary operation $\odot$ on the real unit interval $[0,1]$ that is associative, commutative, with neutral element 1 , and in both arguments monotone. In other words, t-norms are the operations making $[0,1]$ into a (commutative, integral) tomonoid. Moreover, a tnorm $\odot$ is called left-continuous if $\lim _{x}{ }_{a} x \odot b=a \odot b$ for any $a \in(0,1]$ and $b \in[0,1]$. Hence $\odot:[0,1]^{2} \rightarrow[0,1]$ is a left-continuous t-norm if and only if $([0,1] ; \leqslant, \odot, 1)$ is a (commutative, unital) quantale if and only if $([0,1] ; \leqslant, \odot, 1)$ is a (commutative, integral) residuated poset if and only if $([0,1] ; \leqslant, \odot, 1)$ is a (commutative, integral) cut-continuous tomonoid.

Let us consider the four-element Łukasiewicz chain; let $L_{4}=\{-3,-2,-1,0\}$ be endowed with the natural order and the truncated addition $\oplus$ :

$$
d \oplus e=(d+e) \vee-3, \quad d, e \in L_{4},
$$

where + on the right side is the usual addition of integers. Clearly, $\left(L_{4} ; \leqslant, \oplus, 0\right)$ is a tomonoid. Let us moreover consider the tomonoid $\left(\mathbb{R}^{-} ; \leqslant,+, 0\right)$, the negative reals (including 0 ) endowed with the natural order and the usual addition of reals.

We intend to determine totally ordered coextensions of $L_{4}$ by $\mathbb{R}^{-}$, that is, cut-continuous tomonoids $L$ possessing a filter $C$ isomorphic to $\mathbb{R}^{-}$such that $C$ induces a quotient isomorphic to $L_{4}$.

Our tactic is to presuppose the order type of the four $C$-classes. Let us assume that the $C$-class of -3 will be a singleton and the $C$-classes of -2 and -1 will be, like the $C$-class of 0 , order-isomorphic to $\mathbb{R}^{-}$. Thus the universe of the coextension will be

$$
L=\{(-3,0)\} \dot{\cup}\left\{(d, r): d \in\{-2,-1,0\}, r \in \mathbb{R}^{-}\right\},
$$

ordered lexicographically. Note that $L$ itself is order-isomorphic to the real unit interval. Hence, if $L$ can be made into a cut-continuous tomonoid as desired, it will give rise to a left-continuous t-norm.

By a real interval, we will mean any non-empty convex subset of $\mathbb{R}$. We will next see that real intervals can be made into order-transitive $\mathbb{R}^{-}$-modules in essentially only one way: by means of the usual addition of reals.

Lemma 6.3 Let $M$ be an order-transitive $\mathbb{R}^{-}$-module and assume that $M$ is orderisomorphic to a real interval consisting of more than one element. Then there is an order 
isomorphism ı from $M$ to one of $\mathbb{R}^{-}, \mathbb{R}, \mathbb{R}^{+}$, or $[u, 0]$, where $u<0$, such that the action is given by

$$
a \star x=\iota^{-1}(\iota(x)+a)
$$

in the cases that $M$ is not lower bounded, and otherwise by

$$
a \star x=\iota^{-1}((\iota(x)+a) \vee l),
$$

where $l$ is the left endpoint of the real interval $\iota(M)$.

Proof The action $\star: \mathbb{R}^{-} \times M \rightarrow M$ is assumed to be in both arguments cut-continuous. It follows that $\star$ preserves in both arguments the suprema of non-empty upper-bounded subsets.

Fix now some $m \in M$. For any $a, b \in \mathbb{R}^{-}$such that $a \leqslant b, a \star m=b \star m$ implies $b \star m=(a-b) \star(b \star m)$. Consequently, if $a<b$, we have $b \star m=r \star(b \star m)$ for any $r \in \mathbb{R}^{-}$ and, by order-transitivity, $b \star m$ is the smallest element. We distinguish the following cases.

Case 1. $\quad M$ is not bounded from below. Then $\iota: \mathbb{R}^{-} \rightarrow \downarrow m, a \mapsto a \star m$ is injective.

Case 2. $\quad M$ possesses the smallest element $s$. By order-transitivity, $s=a \star m$ for some $a \in \mathbb{R}^{-}$, and since $\star$ preserves non-empty suprema in the first argument, there is a largest element $t \in \mathbb{R}^{-}$with this property. Furthermore, $\iota:[t, 0] \rightarrow \downarrow m, a \mapsto a \star m$ is injective.

We claim that the mapping $\iota$ is surjective in either case. Indeed, assume that there is an $x \in M$ such that $x<m$ and $x$ is not the bottom element of $M$ and such that $a \star m \neq x$ for any $a \in \mathbb{R}^{-}$. Then there is some $b \in \mathbb{R}^{-}$such that $b \star m<x$ but $a \star m>x$ for any $a>b$. But then $c \star x \leqslant b \star m$ for any $c<0$ and hence $x=0 \star x \leqslant b \star m$, a contradiction.

We conclude that, by means of $\iota$, we can identify $\downarrow m$ either with $\mathbb{R}^{-}$or with $[t, 0], t<0$. Furthermore, under this identification, the action is the usual addition of reals. Since the choice of $m \in M$ is arbitrary, the lemma follows.

By Lemma 6.3, the chain $\mathbb{R}^{-}$can be made into an order-transitive $\mathbb{R}^{-}$-module essentially only by means of the usual addition of reals. This observation determines the multiplication in $L$ in the cases where an element of the filter $C=\{0\} \times \mathbb{R}^{-}$is involved: $(d, r) \cdot(0, s)=$ $(d, r+s)$, where $d=-2,-1,0$. Since the class of -3 is a singleton, it is furthermore clear that $(d, r) \cdot(e, s)=(-3,0)$ if $d \oplus e=-3$.

It remains to define the multiplication for pairs of elements of $\{-1\} \times \mathbb{R}^{-}$. We need to determine a suitable operation $\diamond: \mathbb{R}^{-} \times \mathbb{R}^{-} \rightarrow \mathbb{R}^{-}$such that $(-1, r) \cdot(-1, s)=(-2, r \diamond s)$. But the operation $\diamond$ must be a bimorphism of $\mathbb{R}^{-}$-modules; indeed, we must have $(r+t) \diamond s=$ $r \diamond(s+t)=(r \diamond s)+t$ for any $r, s, t \in \mathbb{R}^{-}$. Putting $\sigma=0 \diamond 0 \in \mathbb{R}^{-}$, we conclude

$$
r \diamond s=r+s+\sigma, \quad r, s \in \mathbb{R}^{-} .
$$

It is not difficult to verify that $(L ; \leqslant, \oplus,(0,0))$ defined in this way is, for each choice of $\sigma$, indeed a quantale. In particular, it gives rise to a left-continuous t-norm. Corresponding to the case $\sigma=0$, we show in Fig. 1 how this t-norm may look like.

In Fig. 1 as well as in the subsequent figures, we show the Cayley representation of the tomonoid in question, which consists of the (left, right) inner translations [3, 18]. Furthermore, examples of t-norms are, in order to save space, specified only partly; the definitions can be completed by means of the commutativity condition.

Our example shows how coextensions of cut-continuous tomonoids can be determined in a "modular" way. In general, let $K$ and $C$ be cut-continuous tomonoids. In order to construct the coextensions $L$ of $K$ by $C$, we first assign to each element of $K$ a $C$-module; to the 


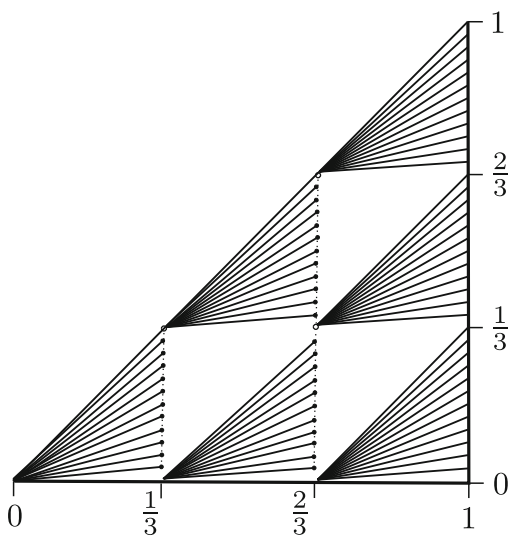

$a \odot b=$
$\left\{\begin{array}{l}a(3 b-2) \\ 3 a b-2 a-b+1 \\ 3 a b-2 a-2 b+2 \\ 0 \\ 3 a b-a-b+\frac{1}{3}\end{array}\right.$

if $a \leqslant \frac{1}{3}$ and $b>\frac{2}{3}$,

if $\frac{1}{3}<a \leqslant \frac{2}{3}$ and $b>\frac{2}{3}$,

if $a, b>\frac{2}{3}$,

if $a \leqslant \frac{1}{3}$ and $b \leqslant \frac{2}{3}$,

if $\frac{1}{3}<a, b \leqslant \frac{2}{3}$

Fig. 1 The t-norm $\odot:[0,1]^{2} \rightarrow[0,1]$ resulting from a coextension of $L_{4}$ by $\mathbb{R}^{-}$

top element 1 we assign $C$ itself, which takes over the role of the congruence-inducing filter. In this way, we get a monoidal operation on $L$ between those pairs of elements one of which is an element of the filter $C$. To extend the multiplication to the remaining $C$-classes, bimorphisms between the $C$-modules need to be determined.

We will not touch the problem of determining all $C$-modules for a given cut-continuous pomonoid $C$. This would amount to checking if there is a free such object, in analogy to the case of modules over quantales $[12,16]$. But we may note that in certain cases, for instance for the negative real cone, there is a quite manageable amount of possibilities. Furthermore, in the general case, the possibilities can be much restricted by presupposing the partial order of the coextended tomonoid. Here, we will rather consider the bimorphisms between given $C$-modules, to which the next section is devoted.

\section{Tensor product of $C$-modules}

Given modules $R, S, T$ over some cut-continuous pomonoid $C$, we are interested in determining all the bimorphisms $\psi: R \times S \rightarrow T$. A tensor product facilitates this task, allowing us to focus on morphisms instead.

Definition 7.1 Let $C$ be a cut-continuous pomonoid and let $R, S$ be $C$-modules. By a tensor product of $R$ and $S$, we mean a bimorphism $\pi$ from $R \times S$ to a further $C$-module denoted by $R \otimes_{C} S$, such that for any bimorphism $\psi$ from $R \times S$ to a $C$-module and sup-lattice $T$ there is a morphism $\tilde{\psi}: R \otimes_{C} S \rightarrow T$ such that $\psi=\tilde{\psi} \circ \pi$.

We shall show that the tensor product of $C$-modules, defined in this specific way, indeed exists. Let us first have a look at the order-theoretical aspects. Erné and Picado introduced in [9] the tensor product of arbitrary closure spaces, fulfilling a universal property for mappings from products of closure spaces that are continuous in each argument. Specialised to the present context, where we deal with the closure operator $\uparrow \downarrow$ on posets, the situation is as follows.

In the sequel, all posets are viewed as closure spaces by means of the respective closure operator $^{\uparrow \downarrow}$. For posets $A$ and $B$, a tensor is a set $W \subseteq A \times B$ such that 
for each $x \in A$, the set $x W=\{y:(x, y) \in W\}$ is a cut in $B$, for each $y \in B$, the set $W y=\{x:(x, y) \in W\}$ is a cut in $A$.

Equivalently we may say that $X \times Y \subseteq W$ implies $X^{\uparrow \downarrow} \times Y^{\uparrow \downarrow} \subseteq W$. The set of all tensors is denoted by $A \otimes B$ and called the tensor product of $A$ and $B . A \otimes B$ is closed under arbitrary intersections and hence makes $A \times B$ into a closure space as well.

For $x, y \in W$, the least tensor containing $(x, y)$ is

$$
x \otimes y=\left(\{x\}^{\downarrow} \times\{y\}^{\downarrow}\right) \cup\left(\varnothing^{\uparrow \downarrow} \times B\right) \cup\left(A \times \varnothing^{\uparrow \downarrow}\right),
$$

called a pure tensor. Note that any tensor $W$ is a union of pure tensors; indeed, we have $W=\bigcup_{(x, y) \in W} x \otimes y$.

We can now formulate a result due to Erné as follows [5, Thm. 3.1] (cf. [9, Thm. 3.3]).

Theorem 7.2 Let $A$ and $B$ be posets. Then the pure tensor insertion

$$
\otimes: A \times B \rightarrow A \otimes B,(x, y) \mapsto x \otimes y
$$

is universal among all separately continuous maps from $A \times B$ to sup-lattices: a map $f$ from $A \times B$ to a sup-lattice $C$ is separately continuous if and only if there is a unique joinpreserving map $\tilde{f}: A \otimes B \rightarrow C$ such that $f(x, y)=\tilde{f}(x \otimes y)$. This map is given by $\tilde{f}(W)=\bigvee f(W)$.

Moreover we have the following results [5, Cor. 4.4, Cor. 4.5] or [9, Cor. 3.1, Thm. 3.2].

Theorem 7.3 Tensor products of posets satisfy the commutative law $A \otimes B \cong B \otimes A$ and the associative law $(A \otimes B) \otimes C \cong A \otimes(B \otimes C)$.

Theorem 7.4 Let $A$ and $B$ be posets. Then $A \otimes B$ and $M C(A) \otimes M C(B)$ are isomorphic sup-lattices, via the bijection

$$
h: A \otimes B \rightarrow \operatorname{MC}(A) \otimes \boldsymbol{M C}(B), W \mapsto\{(X, Y) \in \boldsymbol{M C}(A) \times \boldsymbol{M C}(B): X \times Y \subseteq W\} .
$$

Before continuing with our construction of a tensor product of $C$-modules we will shortly describe sup-lattice coequalisers in $\mathcal{S U} \mathcal{P}$ following [11, Chapter I, Prop. 3].

Let $M$ be a sup-lattice and let $R$ be a binary relation on $M$. Call an element $m \in M R$ coherent if for every $a R b$ we have that $a \leqslant m$ iff $b \leqslant m$. Then the set $M_{R}$ of $R$-coherent elements is a quotient of $M$, the quotient map being

$$
z_{R}: M \rightarrow M_{R}, c \mapsto \bigwedge\{m \in M: m \text { is } R \text {-coherent and } c \leqslant m\}
$$

$z_{R}$ is left adjoint to the inclusion and thus preserves arbitrary joins. It follows that the category of sup-lattices has coequalisers. Indeed, for any

$$
N \stackrel{f}{\stackrel{g}{\longrightarrow}} M
$$

to be coequalised, take $R=\{(f(n), g(n)): n \in N\} \subseteq M \times M$.

We are now in a position to construct the tensor product of $C$-modules $R$ and $S$. We first put $m_{R}: R \times C \rightarrow R,(r, c) \mapsto c \star r$ and $m_{S}: C \times S \rightarrow S,(c, s) \mapsto c \star s$. Since $m_{R}$ and $m_{S}$ are separately cut-continuous, there are by Theorem 7.2 unique join-preserving maps $\tilde{m}_{R}: R \otimes C \rightarrow \mathbf{M C}(R)$ and $\tilde{m}_{S}: C \otimes S \rightarrow \mathbf{M C}(S)$ such that $m_{R}(r, c)=\tilde{m}_{R}(r \otimes c)$ and 
$m_{S}(c, s)=\tilde{m}_{S}(c \otimes s)$ for any $r \in R, c \in C$, and $s \in S$. Taking into account Theorems 7.3 and 7.4, we then have the following pair of morphisms in $\mathcal{S U P}$ :

$$
R \otimes C \otimes S \underset{\operatorname{id}_{R} \otimes \tilde{m}_{S}}{\stackrel{\tilde{m}_{R} \otimes \mathrm{id}_{S}}{\longrightarrow}} R \otimes S .
$$

Let now $g: R \otimes S \rightarrow R \otimes_{C} S$ be the coequaliser for the diagram (4) in $\mathcal{S U P}$ and put $\pi: R \times S \rightarrow R \otimes_{C} S,(r, s) \mapsto g(r \otimes s)$. On $R \otimes_{C} S$, we define a $C$-action as follows. Let $a \in C$; then we let $a \circledast-$ be the join-preserving map from $R \otimes_{C} S$ to itself such that

$$
a \circledast \pi(r, s)=g((a \star r) \otimes s)=g(r \otimes(a \star s))
$$

for any $(r, s) \in R \times S$.

Theorem 7.5 Let $C$ be a cut-continuous pomonoid and let $R, S$ be $C$-modules. Then $R \otimes_{C} S$, made into a $C$-module by Eq. 5, together with $\pi: R \times S \rightarrow R \otimes_{C} S$ is the tensor product of $R$ and $S$.

Moreover, $R \otimes_{C} S$ is also an MC(C)-module, which is isomorphic to the quantale module $M C(R) \otimes_{M C(C)} M C(S)$.

Proof $\pi$ is separately cut-continuous because so is $R \times S \rightarrow R \otimes S, \quad(r, s) \mapsto r \otimes s$ and $g$ is join-preserving. Moreover, $\pi$ preserves the $C$-module action because, for any $a \in C, r \in R$, and $s \in S$, we have $a \circledast \pi(r, s)=\pi(a \star r, s)=\pi(r, a \star s)$. Finally, the universal property of the tensor product follows by the coequaliser universality.

The last assertion is an immediate consequence of Theorem 7.4 and Proposition 2.1: the diagram (4) and the following diagram

$$
\mathbf{M C}(R) \otimes \mathbf{M C}(C) \otimes \mathbf{M C}(S) \underset{\operatorname{id}_{\mathbf{M C}(R)} \otimes \tilde{m}_{\mathbf{M C}(S)}}{\stackrel{\tilde{m}_{\mathbf{M C}(R)} \otimes \operatorname{id}_{\mathbf{M C}(S)}}{\longrightarrow}} \mathbf{M C}(R) \otimes \mathbf{M C}(S) .
$$

coincide up to isomorphism.

In order to be able to apply Theorem 7.5 to the determination of bimorphisms between $C$-modules that are not necessarily sup-lattices, we shall make use of the fact that a $C$-action on a poset $R$ can be extended to an action on the Dedekind-MacNeille completion $\mathbf{M C}(R)$.

Proposition 7.6 Let $C$ be a cut-continuous pomonoid and let $R$ be a $C$-module. Identifying $R$ with its image under $e_{R}: R \rightarrow M C(R)$, we can extend the action on $R$ to an action on $M C(R)$, making the latter into a $C$-module as well.

Proof By Proposition 2.1, each morphism $a \star-: R \rightarrow R$ possesses a unique extension to a join-preserving map $a \star-: \mathbf{M C}(R) \rightarrow \mathbf{M C}(R)$. In particular, $\star$ is cut-continuous in the second argument. To see that $\star$ is cut-continuous in the first argument, let $x, y \in \mathbf{M C}(R)$. Then $x=\bigvee_{\iota \in I} x_{\iota}$ and $y=\bigwedge_{\kappa \in K} y_{\kappa}$ for some $x_{\iota}, y_{\kappa} \in R$ (where $I, K$ are possibly empty) and it follows that

$$
\{a \in C: a \star x \leqslant y\}=\bigcap_{\iota, \kappa}\left\{a \in C: a \star x_{\iota} \leqslant y_{\kappa}\right\}
$$

is a cut in $C$. Finally, the fact that $R$ is join-dense in $\mathbf{M C}(R)$ also implies that $\star$ is still a module action. 
Assume now that we are given $C$-modules $R, S$, and $T$ and we want to determine the bimorphisms from $R \times S$ to $T$. Then for any bimorphism $f: R \times S \rightarrow T$ there is a morphism $\tilde{f}: R \otimes_{C} S \rightarrow \mathbf{M C}(T)$ such that $e_{T} \circ f=\tilde{f} \circ \pi$. Consequently, by determining the morphisms from $R \otimes_{C} S$ to $\mathbf{M C}(T)$, we get all desired bimorphisms.

In order to find all bimorphisms from $R \times S$ to $T$ we may thus proceed as follows. (1) We determine the morphisms $\tilde{f}: R \otimes_{C} S \rightarrow \mathbf{M C}(T)$. (2) For each such morphism $\tilde{f}$, we check whether $\tilde{f} \circ \pi$ maps $R \times S$ to $T$, viewed as a subset of $\mathbf{M C}(T)$.

We note that step (2) cannot easily be avoided. As our approach relies on the tensor product of closure spaces, we defined the tensor product to have the universal property only with regard to morphisms to modules that are also sup-lattices. An alternative way seems not to be straightforward.

\section{An Example of a Coextension}

We shall discuss a further example from the realm of left-continuous t-norms. We note that for this example we could certainly determine the necessary bimorphisms without difficulties directly. However, our intention is to put the construction into the wider context given by the framework that we have developed above.

Let $K$ be the finite tomonoid depicted in Fig. 2. We wish to coextend $K$ by an Archimedean cut-continuous tomonoid such that the result $L$ gives rise to a left-continuous t-norm. Then the tomonoid by which we coextend $K$ must either be isomorphic to the negative cone $\mathbb{R}^{-}$or the negative unit interval $[-1,0]$, endowed with the (truncated) usual addition of reals [18, Prop. 6.3]. Let us discuss the former case: we will use the tomonoid $\left(\mathbb{R}^{-} ; \leqslant,+, 0\right)$.

Since the congruence classes will by assumption be order-isomorphic to real intervals as well, the required order-transitive $\mathbb{R}^{-}$-modules are by Lemma 6.3 (up to isomorphism) real intervals upon which the elements of $\mathbb{R}^{-}$act by usual addition. The only necessary choice concerns the type of interval: a singleton, $\mathbb{R}^{-},[u, 0]$ for some $u<0, \mathbb{R}^{+}$, or $\mathbb{R}$.

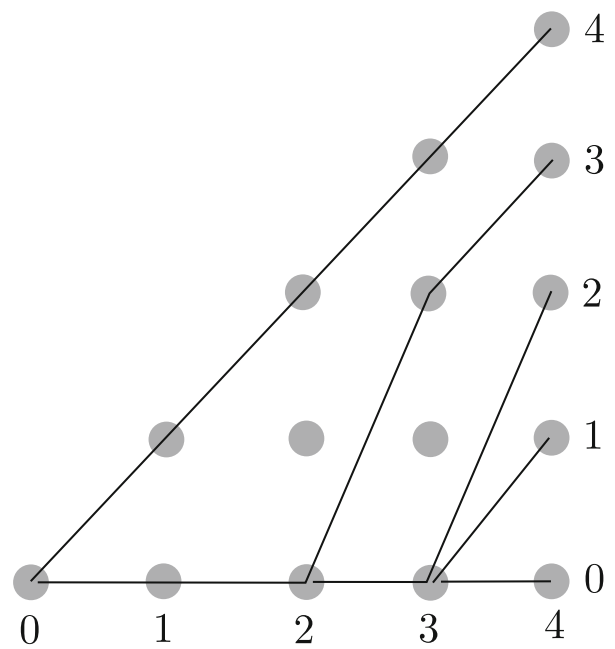

Fig. 2 The tomonoid $K$ 
We shall use, from bottom to top, $\mathbb{R}^{-}$-modules based on $\mathbb{R}^{+},\{0\}, \mathbb{R}^{-}, \mathbb{R}^{-}$, and $\mathbb{R}^{-}$. (The top-most interval must of course be chosen in accordance with the tomonoid by which we coextend.) Hence

$$
L=\{0\} \times \mathbb{R}^{+} \dot{U}\{(1,0)\} \dot{U}\left\{(d, r): d \in\{2,3,4\}, r \in \mathbb{R}^{-}\right\},
$$

endowed with the lexicographic order. Here, $C=\{4\} \times \mathbb{R}^{-}$will be the congruence-inducing filter. The multiplication between elements one of which is from $C$ is given by the action of $C$ on the respective class. Furthermore, the multiplication with the single element of the class $\{(1,0)\}$ is trivial, leading always to the bottom element of the respective class. In order to determine the multiplication between the remaining elements, we shall calculate the necessary tensor products; we need to determine $\mathbb{R}^{-} \otimes_{\mathbb{R}^{-}} \mathbb{R}^{-}$and $\mathbb{R}^{-} \otimes_{\mathbb{R}^{-}} \mathbb{R}^{+}$. We shall write $\overline{\mathbb{R}^{-}}=\mathbf{M C}\left(\mathbb{R}^{-}\right)=\mathbb{R}^{-} \cup\{-\infty\}$, where $-\infty$ denotes the bottom element, and similarly $\overline{\mathbb{R}^{+}}=\mathbf{M C}\left(\mathbb{R}^{+}\right)=\mathbb{R}^{+} \cup\{\infty\}$.

The tensor product of posets $\mathbb{R}^{-} \otimes \mathbb{R}^{-}$consists of the sets $\left\{(x, y) \in \mathbb{R}^{-} \times \mathbb{R}^{-}: y \leqslant\right.$ $\tau(x)\}$, where $\tau$ is an antitone left-continuous map from $\mathbb{R}^{-}$to $\overline{\mathbb{R}^{-}}$(that is, a map from $\mathbb{R}^{-}$ to the dual of $\overline{\mathbb{R}^{-}}$preserving non-empty suprema). As a sup-lattice, $\mathbb{R}^{-} \otimes \mathbb{R}^{-}$is generated by $r \otimes s=\{(x, y): x \leqslant r, y \leqslant s\}, r, s \in \mathbb{R}^{-}$. The coequaliser in $\mathcal{S U} \mathcal{P}, g: \mathbb{R}^{-} \otimes \mathbb{R}^{-} \rightarrow$ $\mathbb{R}^{-} \otimes_{\mathbb{R}^{-}} \mathbb{R}^{-}$, is required to fulfil $g((r+c) \otimes s)=g(r \otimes(s+c))$ for all $c \in \mathbb{R}^{-}$. This means that $g(r \otimes s)=g(0 \otimes(r+s))$, hence $g(r \otimes s)=g\left(r^{\prime} \otimes s^{\prime}\right)$ whenever $r+s=r^{\prime}+s^{\prime}$. We conclude that we can put $\mathbb{R}^{-} \otimes_{\mathbb{R}^{-}} \mathbb{R}^{-}=\overline{\mathbb{R}^{-}}$and $g(r \otimes s)=r+s$. The $\mathbb{R}^{-}$-action on $\overline{\mathbb{R}^{-}}$is the usual the addition of reals: for $c \in \mathbb{R}^{-}$, we have $c \circledast x=x+c$ if $x \in \mathbb{R}^{-}$and $c \circledast-\infty=-\infty$.

Similarly, the tensor product $\mathbb{R}^{-} \otimes \mathbb{R}^{+}$consists of $\left\{(x, y) \in \mathbb{R}^{-} \times \mathbb{R}^{+}: y \leqslant \tau(x)\right\}$, where $\tau$ is an antitone left-continuous map from $\mathbb{R}^{-}$to $\overline{\mathbb{R}^{+}}$. This time the coequaliser $g$ has to fulfil $g((r+c) \otimes s)=g(r \otimes(s+c) \vee 0)$ for all $c \in \mathbb{R}^{-}$, where $r \in \mathbb{R}^{-}$and $s \in \mathbb{R}^{+}$. Thus $g(r \otimes s)=g(0 \otimes(r+s) \vee 0)$ and hence $g(r \otimes s)=g\left(r^{\prime} \otimes s^{\prime}\right)$ whenever $(r+s) \vee 0=\left(r^{\prime}+s^{\prime}\right) \vee 0$. We can put $\mathbb{R}^{-} \otimes_{\mathbb{R}^{-}} \mathbb{R}^{+}=\overline{\mathbb{R}^{+}}$and $g(r \otimes s)=(r+s) \vee 0$. The $\mathbb{R}^{-}$-action on $\overline{\mathbb{R}^{+}}$is, for $c \in \mathbb{R}^{-}$, given by $c \circledast x=(x+c) \vee 0$ if $x \in \mathbb{R}^{+}$and $c \circledast \infty=\infty$.

We next have to determine the morphisms between the $\mathbb{R}^{-}$-modules $\overline{\mathbb{R}^{-}}$and $\overline{\mathbb{R}^{+}}$. We readily see that a morphism $h: \overline{\mathbb{R}^{-}} \rightarrow \overline{\mathbb{R}^{-}}$is of the form

$$
h(x)= \begin{cases}x+\sigma & \text { if } x \in \mathbb{R}^{-} \\ -\infty & \text { if } x=-\infty\end{cases}
$$

where $\sigma \leqslant 0$. Moreover, for a morphism $h: \overline{\mathbb{R}^{-}} \rightarrow \overline{\mathbb{R}^{+}}$we have

$$
h(x)= \begin{cases}(x+\sigma) \vee 0 & \text { if } x \in \mathbb{R}^{-}, \\ 0 & \text { if } x=-\infty\end{cases}
$$

where $\sigma \geqslant 0$. Finally, a morphism $\overline{\mathbb{R}^{+}} \rightarrow \overline{\mathbb{R}^{+}}$is of the form

$$
h(x)= \begin{cases}(x+\sigma) \vee 0 & \text { if } x \in \mathbb{R}^{+}, \\ \infty & \text { if } x=\infty\end{cases}
$$

where $\sigma \leqslant 0$. 


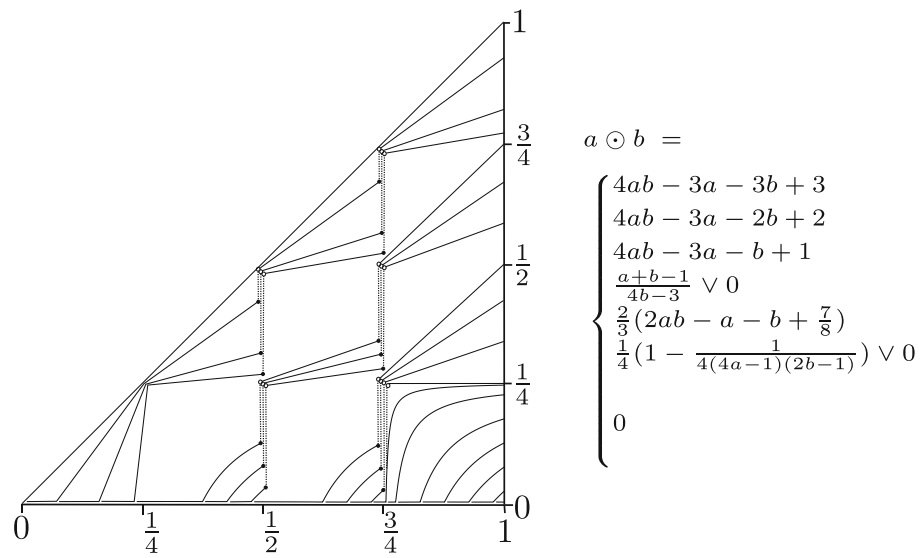

$$
\begin{aligned}
& \text { if } a, b>\frac{3}{4}, \\
& \text { if } b>\frac{3}{4} \text { and } \frac{1}{2}<a \leqslant \frac{3}{4}, \\
& \text { if } b>\frac{3}{4} \text { and } \frac{1}{4}<a \leqslant \frac{1}{2}, \\
& \text { if } b>\frac{3}{4} \text { and } a \leqslant \frac{1}{4}, \\
& \text { if } \frac{1}{2}<a, b \leqslant \frac{3}{4}, \\
& \text { if } \frac{1}{2}<b \leqslant \frac{3}{4} \\
& \text { and } \frac{1}{4}<a \leqslant \frac{1}{2}, \\
& \text { if } b \leqslant \frac{3}{4} \text { and } a \leqslant \frac{1}{4}, \\
& \text { or } a, b \leqslant \frac{1}{2} .
\end{aligned}
$$

Fig. 3 Example of a t-norm $\odot$ arising from the coextension of $K$ by $\mathbb{R}^{-}$

We conclude that any integral coextension of $K$ by $\mathbb{R}^{-}$is given according to the following prescriptions:

$$
\begin{aligned}
& (4, r) \odot(4, s)=(4, r+s), \\
& (4, r) \odot(3, s)=(3, r+s), \\
& (4, r) \odot(2, s)=(2, r+s), \\
& (4, r) \odot(1,0)=(1,0), \\
& (4, r) \odot(0, s)=(0,(r+s) \vee 0), \\
& (3, r) \odot(3, s)=\left(2, r+s+\sigma_{1}\right), \quad \text { where } \sigma_{1} \leqslant 0, \\
& (3, r) \odot(2, s)=\left(0,\left(r+s+\sigma_{2}\right) \vee 0\right), \quad \text { where } \sigma_{2} \geqslant 0, \\
& (a, r) \odot(b, s)=(0,0) \quad \text { for any } a \leqslant 3, b \leqslant 1 \text { or } a=b=2 ;
\end{aligned}
$$

here $r, s \in \mathbb{R}$ are such that the indicated pairs are, respectively, in $L$.

It is easily checked that we define in this way for any choice of $\sigma_{1}$ and $\sigma_{2}$ indeed a residuated tomonoid. After rescaling we get a cut-continuous t-norm; for an example based on a choice of $\sigma_{1}<0$ and $\sigma_{2}>0$, see Fig. 3 .

\section{Conclusion}

Cut-continuous pomonoids generalise residuated posets; for any pair of elements $x, y$, the sets $\{c: y \cdot c \leqslant x\}$ and $\{c: c \cdot y \leqslant x\}$ are no longer required to be principal ideals but just cuts. Cut-continuous pomonoids naturally lead to two different categories. In $q \mathcal{C C M}$, the morphisms are chosen in analogy to the case of quantales. The Dedekind-MacNeille completion gives rise to a reflector from $q \mathcal{C C M}$ to the category of unital quantales. In contrast, morphisms are chosen in $r \mathcal{C C M}$ in such a way that the case of residuated lattices is generalised.

Any congruence, defined accordingly, is then induced by a filter, in the same way as in the case of residuated lattices. Our interest has focused on the question how to construct coextensions of some cut-continuous pomonoid $K$ by a further one $C$. To this end, all possible $C$-modules as well as the bimorphisms between them need to be determined. 
The latter need has motivated us to study tensor products of modules over cut-continuous pomonoids. Whereas in the context of residuated lattices there is not much to say here, the present context is surely more profitable. Elaborating on results of M. Erné and J. Picado on closure spaces, we have shown a tensor product to exist. We have demonstrated the role that the tensor product plays in the construction of coextensions by means of an example originating from fuzzy logic: the construction of left-continuous t-norms.

Several problems remained open and should be addressed in further research. First, our results apply to the construction of coextensions in the case of a total order, whereas the general case remains tricky; the problem is that the restriction of the monoidal operation to a pair of congruence classes is seen to be cut-continuous only under quite special assumptions. Furthermore, following Erné and Picado's approach, the formation of tensor products always leads to a complete structure and hence the correspondence between the bimorphisms and the morphisms of the tensor product is not one-to-one. A progress in this respect would require, however, a different approach.

Acknowledgments Open access funding provided by Austrian Science Fund (FWF). The authors acknowledge the support by the Czech Science Foundation (GAČR): project 15-34697L and the Austrian Science Fund (FWF): project I 1923-N25.

They would moreover like to express their gratitude to the anonymous reviewers whose thorough and detailed comments lead to a considerable improvement of this paper. Finally, the authors are indepted to M. Erné whose article [8] helped to enhance the results of this paper in a substantial manner.

Open Access This article is distributed under the terms of the Creative Commons Attribution 4.0 International License (http://creativecommons.org/licenses/by/4.0/), which permits unrestricted use, distribution, and reproduction in any medium, provided you give appropriate credit to the original author(s) and the source, provide a link to the Creative Commons license, and indicate if changes were made.

\section{References}

1. Bishop, A.: A universal mapping characterization of the completion by cuts. Algebra Univers. 8, 349353 (1978)

2. Blount, K., Tsinakis, C.: The structure of residuated lattices. Int. J. Algebra Comput. 13, 437-461 (2003)

3. Clifford, A.H., Preston, G.B.: The algebraic theory of semigroups, vol. I. American Mathematical Society, Providence (1961)

4. Erné, M.: On the relativization of chain topologies. Pacific. J. Math. 84, 43-52 (1979)

5. Erné, M.: Tensor products for bounded posets revisited. Order 7, 295-314 (1990)

6. Erné, M.: The Dedekind-MacNeille completion as a reflector. Order 8, 159-173 (1991)

7. Erné, M.: Closure. In: Mynard, F., Pearl, E. (eds.) Beyond topology, Contemporary Mathematics, vol. 486, pp. 163-238. American Mathematical Society, Providence (2009)

8. Erné, M.: Cut-continuity, cut-compatibility, filters and congruences of pomonoids submitted (2018)

9. Erné, M., Picado, J.: Tensor products and relation quantales. Algebra Univer. 78, 461-487 (2017)

10. Erné, M., Reichman, J.: Completions of partially ordered semigroups. Semigroup Forum 34, 253-285 (1987)

11. Joyal, A., Tierney, M.: An extension of the Galois theory of Grothendieck. American Mathematical Society, Providence (1984)

12. Kruml, D.: Spatial quantales. Appl. Categ. Struct. 10, 49-62 (2002)

13. Kruml, D., Paseka, J.: Algebraic and categorical aspects of quantales. In: Handbook of Algebra, volume 5, pp. 323-362, North-Holland (2008)

14. Nelson, E.: Galois connections as left adjoint maps. Commentat. Math. Univ. Carol. 17, 523-541 (1976)

15. Rosenthal, K.I.: Quantales and their applications, Pitman Research Notes in Mathematics. Longman Scientific \& Technical, New York (1990) 
16. Russo, C.: Quantale modules and their operators, with applications. J. Log. Comput. 20, 917-946 (2010)

17. Tsinakis, C., Wille, A.M.: Minimal varieties of involutive residuated lattices. Stud. Log. 83, 407-423 (2006)

18. Vetterlein, T.: Totally ordered monoids based on triangular norms. Commun. Algebra 43, 2643-2679 (2015) 